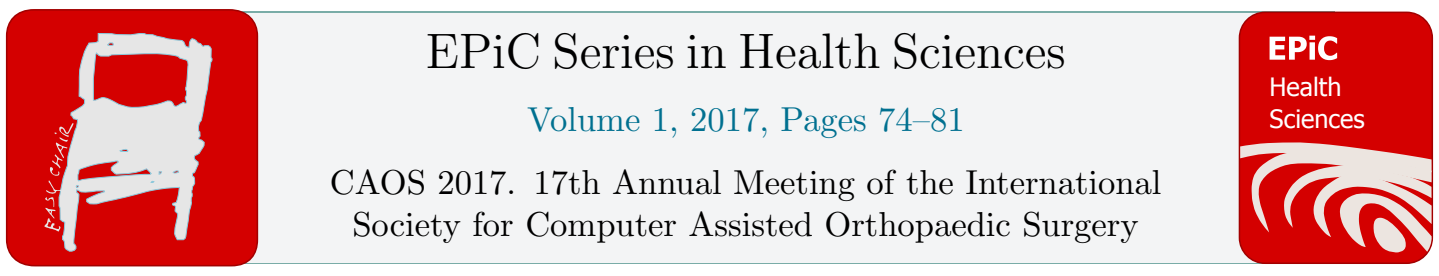

\title{
Individual Cooling and Compression System for Accelerated Swelling Reduction on Fractures
}

\author{
Annekathrin Paessler ${ }^{1}$, Markus Fielitz ${ }^{1}$, Heiko Rathmann ${ }^{1}$, \\ Konstantin Andrusch ${ }^{2}$, Thomas Nagel ${ }^{1}$ and Jens Lienig ${ }^{1}$ \\ ${ }^{1}$ Dresden University of Technology / Institute of Electromechanical and Electronic Design, \\ Dresden, Germany \\ ${ }^{2}$ Dresden University of Technology / Institute of Manufacturing Science and Engineering, \\ Dresden, Germany \\ annekathrin.paessler@tu-dresden. de
}

\begin{abstract}
After a fracture injury a swelling of the skin soft tissue results which prevents an early operation. In order to shorten the waiting time until the decrease of the swelling, the metabolism-reducing effect of mild local hypothermia and the swelling-reducing effect of compression therapy should be used in a device anatomically adjusted to the patient. For the best possible treatment, a regulation of the cooling and compression parameters should be carried out by means of sensor technology in order to allow an adaptation to the individual patient.

Based on medical studies, parameter areas relevant to patients could be determined. These were implemented in first functional models for a cooling system and a compression system. Anatomically adapted cooling and pressure chambers allow a good physical coupling of the system to humans. The first test results, considering the heat emission and the skin elasticity properties of the human body, show that both rapid cooling can be achieved in a range of between 17 and $20^{\circ} \mathrm{C}$ of skin temperature within $4 \mathrm{~min}$, and that under a pressure effect of between 10 and $20 \mathrm{kPa}$ in the pressure chambers a pressure on the human tissue can be produced up to $15 \mathrm{kPa}$. The cooling system based on Peltier elements is capable of transporting a heat flow up to $10 \mathrm{~W}$. The quiet, maintenance-free Peltier elements also increase the patient's acceptance. In the pressure chambers, a temporally overlapping generation of pressure pulses can imitate the process of natural walking by the effect of muscle pumps and contribute to a technical lymphatic drainage. By detecting the inflammation area with the aid of netlike-arranged temperature sensors and measuring the internal pressure of the pressure chambers in the case of a swelling tissue, cooling can be carried out at a determined point and the occurrence of, for example, a compartment syndrome can be prevented by regulating the pressure.
\end{abstract}


At present still ongoing, promising developments of models for the consideration of the temperature distribution and pressure differences in the cardiovascular system should enable a determination of optimized cooling and pressure curves adapted to the individual human. In this way, the best possible control circuits should be identified using medical expert knowledge.

\section{Introduction}

With an incidence of about $1 \%$ [12], the ankle fracture (ICD 10 S82) is one of the most common injuries in traumatology in the European population. If an unstable or shifted fracture exists, surgical treatment must be executed. Shortly after the injury process, a swelling of the skin soft tissues in the fracture area is formed. As soon as this pronounced swelling occurs, an operation of the fracture cannot take place immediately. Hence, operations are delayed until the reduction of the swelling occurs, which may take 7 to 14 days [8]. Increased treatment costs, longer work loss and extended pain periods for the patients are the result. If the preoperative support bandage is additionally insufficiently padded or narrows the limb, complications can occur during swelling. In addition to pressure ulcers and pressure damage to nerves, partial or complete blood flow disturbances may occur. This can lead to a hypoxia, an ischemia or a compartment syndrome.

For prevention or treatment of such a tissue swelling, there are various examined principles. The two most promising principles with their effects are:

- Prevention of swelling formation due to the influence of mild, local hypothermia on the skin surface at the inflammatory area.

- Reduction of a formed swelling by pulsed compression on subcutaneously situated veins and lymphatic vessels to simulate muscle pumps.

A corresponding system with coupling of both influences, adjusted to the individual patient, will be presented below. Systems already placed on the market $[3,5,16]$ partly combine these two influences, but are not adapted to the individual patient by monitoring with sensors and therefore possible regulation.

\section{Materials and Methods}

On the basis of a lot of medical studies, it is useful for an optimal adaptation to the patient to implement a combined controlled cooling and compression in one medical device. As shown in [13], cooling and compression should be integrated into one common system, patient-near pressure chambers and cooling chambers should be adapted to the type of fracture and the anatomical conditions and optimized cooling and pressure curves have to be traversed while measuring individual patient data. The following sections briefly present the individual components.

\subsection{Cooling system}

The cooling system consists of different individual components (see Fig. 1a): A heat exchanger made of four Peltier elements contributes to the cooling of a cooling medium. The cooling medium is transported through a cooling pipe to fracture-near cooling chambers. A targeted cooling is achieved by independently controllable valves for the selection of the corresponding cooling chambers. There the heat is extracted from the body by the temperature difference to the cooling medium. To be able to 
react to local inflammatory areas, distributed temperature sensors are used to measure the skin temperature.

Peltier elements, in contrast to other possibilities of forming a heat exchanger, have many advantages in the field of medical technology, such as a compact, space-saving design, quietness, maintenance- and vibration-freedom due to missing moving parts, low operating voltages, freedom from toxic coolants and the possibility to precisely control the desired temperature. Peltier elements of the type RIME-74 from the company Kryotherm are used. As cooling chambers (see Fig. 1b) no hydraulic hoses are used which are ideally circular in cross section, but a meander-shaped mold is manufactured based on the so-called tubular films. The advantage is the better flat adaptation to the human anatomy due to directed application of the larger surfaces of the film on the skin. Because of the larger contact surface, the heat convection is significantly improved. Polyvinyl chloride, PVC for short, is used for the material selection for the cooling chambers. For a watertight, sufficiently pressure-resistant connection of the two PVC film parts, a simplified thermal contact welding is possible. As a cooling medium, water is used. In order to detect the inflammatory area, sensor arrangements with hot-conducting SMD thermistors with a tolerance of $\pm 1 \%$ at $25^{\circ} \mathrm{C}$ are built.

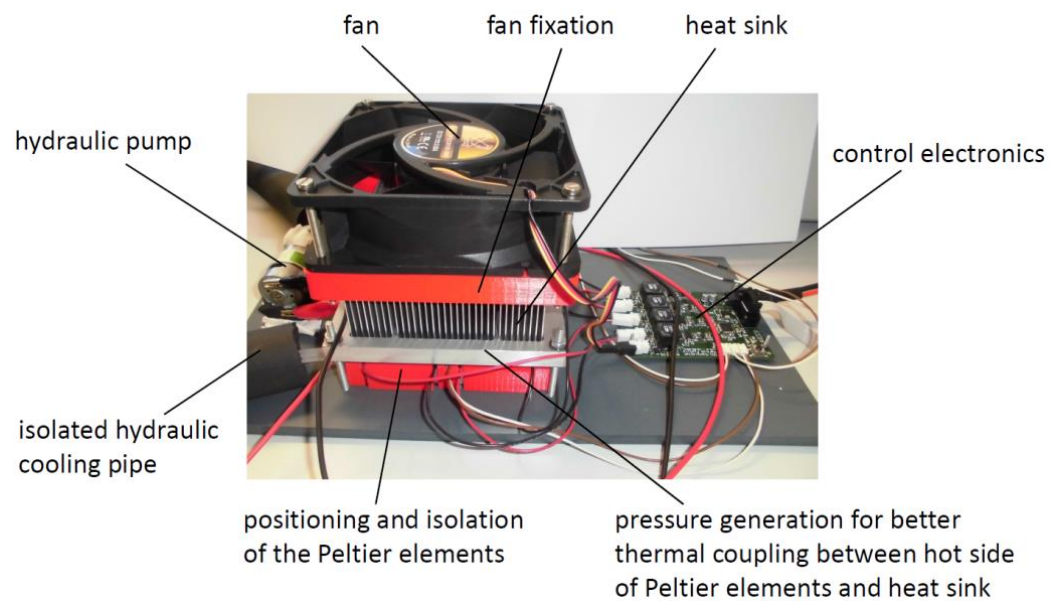

a) Control unit of the cooling system

Figure 1: Structure of the functional model of the cooling system

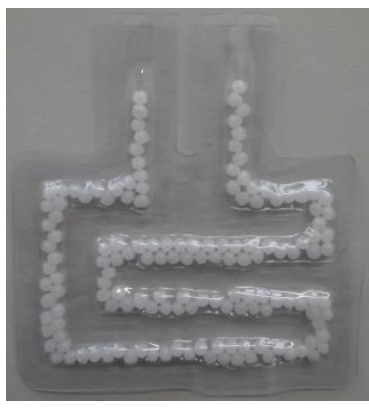

b) Cooling chamber

\subsection{Compression system}

The compression system is also a combination of many individual components (see Fig. 2a). A rough division is as follows: A pneumatic pump directs a pressure medium from the outside into a pneumatic feed line. The switching state of intake valves determines which connected pressure chamber the pressure medium can spread to. The pressure medium can leave the pressure chamber again by outlet valves. If both valve types are closed, a constant pressure is maintained in the pressure chamber, which contributes to the stabilization of the fracture from the outside. If the tissue continues to swell, the resulting pressure change in the chambers can be detected by pressure sensors and a corresponding pressure release is triggered to prevent pressure ulcers. An analogous inflation of the chambers can also occur with swelling decrease. For the technical imitation of manual lymphatic drainage, it is possible to perform the course of peristaltic, temporally overlapping pressure pulses from distal to proximal pressure chambers.

The pressure chambers are located between the swelling skin tissue and an external stabilizing medium. The effect of the technical lymph drainage should be increased and the wearing comfort 
should be improved by a chamber shape adapted to the anatomy of the patient as well as a filling of the chambers with padding. The pressure chambers should be produced from the same material as the cooling chambers, but are currently manufactured from airbag fabrics. As a pressure medium ambient air is used.

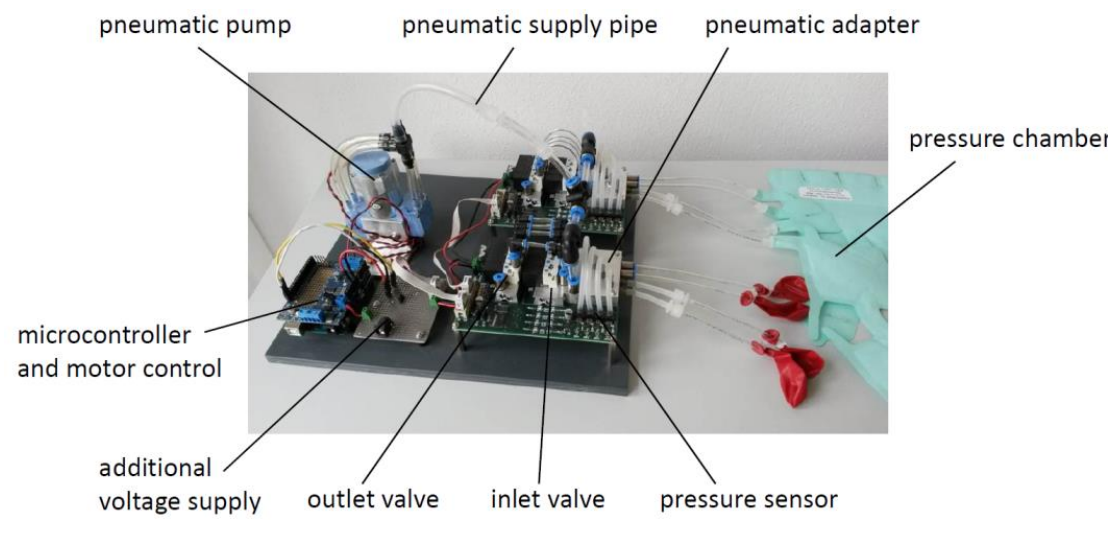

a) Control unit of the compression system

Figure 2: Structure of the functional model of the compression system

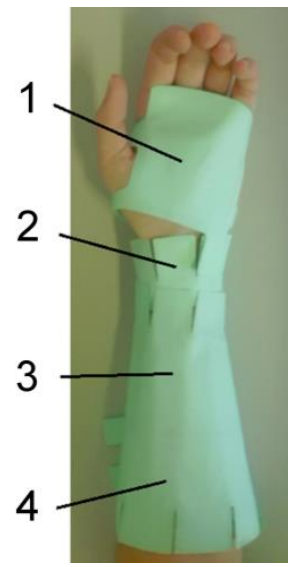

b) Pressure chambers (distal to proximal)

\subsection{Simulation}

A regulation for adaptation to the individual patient should be implemented in the designed cooling and compression system. The goal is to develop an optimized cooling curve as well as a pressure curve adapted to the physiological characteristics of humans. For this reason, a partial model of physiology for simulating the temperature and pressure behavior in the human body is developed. The focus is on the cardiovascular system, which transports blood heated in the internal body or in muscles to the extremity, thereby simultaneously building a pressure gradient in the different blood vessels. In the simulation with the software SimulationX 3.7, the optimized curves can be identified and later integrated into the hardware as parameter related functions. Thus it is possible to detect problems without real patient contact. For an analogous modeling of the human anatomy, an orientation to DIN 33402-2 on the ergonomics of the average male body based on anthropometric measurements is carried out [4]. The generated model of the cardiovascular system is composed of the series circuit of the systemic circulation and the pulmonary circulation. The organs of the systemic circulation can be compared with resistors connected in parallel. For a closer look in this work, a human leg with foot is split up in more detail. The remaining organs and extremities are abstracted.

\section{Results}

\subsection{Cooling system}

In order to characterize the resulting system and determine its performance, many measurements were made under various conditions. For the validation of the system, a heating resistor is installed on one cooling chamber, which is to imitate the heat emission of the human skin. A simple PI regulator is designed for testing. When a heat flow of $2 \mathrm{~W}$ is used in the heating resistor, a regulator sequence is obtained, as can be seen in Fig. 3. The skin temperature should be maintained between 17 and $20^{\circ} \mathrm{C}$ 
[9, 11]. It is obvious that the target temperature is reached after $3.7 \mathrm{~min}$ starting from the room or skin temperature and fluctuates in the further course between the two limit values. According to the temperature on the hot side of the Peltier element, the fan is operated with the duty factor $\mathrm{d}_{\mathrm{CL}}$. In order to understand the importance of this measurement for the application of the cooling system for the swelling treatment, the surface of the cooling chambers must be considered. Its base area $A_{\text {cool }}$ is $70 \mathrm{~cm}^{2}$. According to the Newtonian approach [15], the heat flow $\dot{Q}_{c o o l}$ from the human body results from the skin surface. The heat transfer coefficient $k_{\text {human }}$ of humans is $10 \frac{\mathrm{W}}{\mathrm{m}^{2} \cdot \mathrm{K}}$ on bare skin according to [15]. If a maximum skin temperature of $36^{\circ} \mathrm{C}$ and a minimum cooling medium temperature of $15^{\circ} \mathrm{C}$ are used, a human could give the following maximum heat flow over the surface of the cooling chamber:

$$
\dot{Q}_{\text {cool }}=k_{\text {human }} \cdot A_{\text {cool }} \cdot\left(\vartheta_{\text {human }}-\vartheta_{\text {water }}\right)=1,47 \mathrm{~W}
$$

With a safety factor, this corresponds approximately to the set $2 \mathrm{~W}$. Working at full power, the system can carry a heat flow of $10 \mathrm{~W}$.

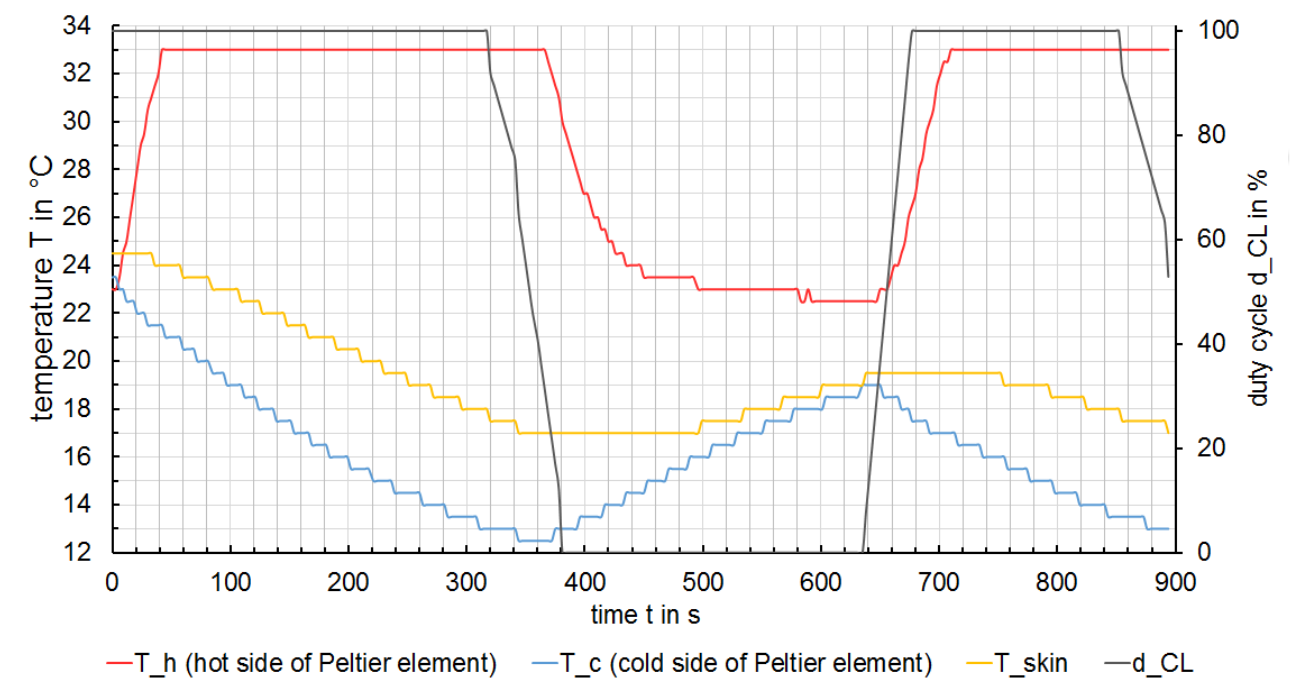

Figure 3: Temperature control with a heat flow of $2 \mathrm{~W}$

\subsection{Compression system}

To measure the effect of the pressure chambers, a comparison was made between the applied pressure in the chamber and the pressure on the human tissue. In order to properly recreate a support bandage, a dressing based on the following layers was developed: gauze bandage, flat FSR 406 sensor, pressure chamber of $50 \times 50 \mathrm{~mm}^{2}$, elastic bandage, inelastic tape. In the compression system, target pressures of $10 \mathrm{kPa}(75 \mathrm{mmHg}), 15 \mathrm{kPa}(113 \mathrm{mmHg})$ and $20 \mathrm{kPa}(150 \mathrm{mmHg})[1,16]$ were determined for three different measurements (see Fig. 4). After reaching the target pressure, a pause of $3 \mathrm{~s}$ was inserted and then inflated again to the desired pressure. It can be recognized that as the target pressure increases, a rising difference to the actual pressure is recorded. Presumably, this is due to the greater air discharge from the non-ideal-tight pressure chamber and the compressibility of the skin tissue. However a considerable increase in the pressure was confirmed by the test person. After each pressure pulse, the pressure drops about $4 \mathrm{kPa}(30 \mathrm{mmHg})$ within the pause time because of the 
short-term hermetic airbag fabrics. Therefore, an equivalent pressure chamber made of PVC, which promises constant pressure to be maintained, must be tested in the next tests. A significant reduction in the pressure after the end of the pressure pulses by approximately $2 \mathrm{kPa}(15 \mathrm{mmHg})$ with respect to the initial pressure at the starting time could be noticed. This demonstrates the good effect of technical lymphatic drainage, which can remove blood and lymphatic fluid from the tissue. Only after about 10 to $20 \mathrm{~s}$ the pressure in the calf has again increased to the initial pressure by inflowing blood.

\subsection{Simulation}

Fig. 5a shows the structure of the individual anatomical components of the cardiovascular system. A usual course of the blood volume in the left ventricle, which was expelled over time, was modeled according to [6]. The curve profile of the simulated compression curve is designed in a good approximation to the desired target curve [14]. The total peripheral resistance of the systemic circulation therefore seems to agree well with reality. Comparisons of the pressure profiles in the large arteries (target: $100 \pm 40 \mathrm{mmHg}$, simulated: $110 \pm 40 \mathrm{mmHg}$ ), the capillaries (target: $30 \mathrm{mmHg}$, simulated: $45 \mathrm{mmHg}$ ) and the large veins (target: $3 \mathrm{mmHg}$, simulated: $0.2 \mathrm{mmHg}$ ) confirm this claim [6]. Through the presence of a model of physiology, it is possible to couple models of the cooling and compression system with it. The cooling system can, for example, be connected to the thermal elements of the heat conduction of the skin (Fig. 5b). The pressure system is coupled with the model of physiology by a piston structure similar to the muscle pumps (Fig. 5c), but additional has pressure sensors and valves. By means of these couplings, it is possible to obtain adjusted, optimized cooling and pressure curves adapted to the human, with the integration of the simulation results into an optimization software.

\section{Discussion}

The aim of this work was to develop a functioning device consisting of a cooling unit and a compression unit, which can prevent a swelling or reduce it as quickly as possible.

The resulting cooling system is based on four Peltier elements. The required skin temperature of 17 to $20^{\circ} \mathrm{C}$ could be achieved after $4 \mathrm{~min}$ in a laboratory test with one cooling chamber heated to a maximum of $10 \mathrm{~W}$. Through the connection of several cooling chambers, a heat flow of $2 \mathrm{~W}$ per cooling chamber corresponding to human physiology can thereby be dissipated. The cooling chambers can be connected via valves, in order to make a cooling possible only at the locations with the focus of inflammation. Thus it is confirmed that Peltier elements are quite suitable for use in a compact, mobile cooling device for the swelling control of fracture tissue. In the future, whether ambulatory, in a hospital car or stationary, this practice could accelerate the healing or allow operations earlier due to a temperature-controlled cooling.

The functional model developed for the section of the compression shows the technical implementation of the lymphatic drainage with an intermittent pneumatic compression therapy. PVC instead of airbag chambers should be used because of the tightness. In hospitals, standardized compressed air could be used via pneumatic ports. This source generates an inexhaustible volume flow.

With regard to the simulation of human physiology, in contrast to previously developed models such as $[2,7,10]$, no separation or sole assessment of hydraulics and thermodynamics is made in independent submodels. The focus is on the interaction of pressure and temperature changes. In addition, the lymphatics are examined in connection with the cardiovascular system as a novelty. 


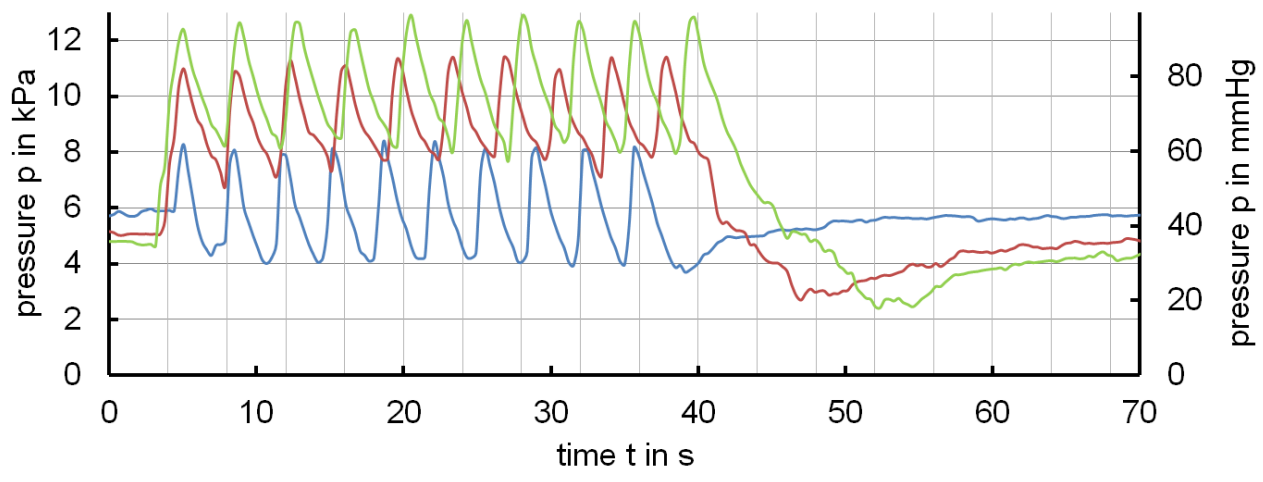

$-10 \mathrm{kPa}-15 \mathrm{kPa}-20 \mathrm{kPa}$

Figure 4: Pressure from a pressure chamber on a human calf with different target pressures

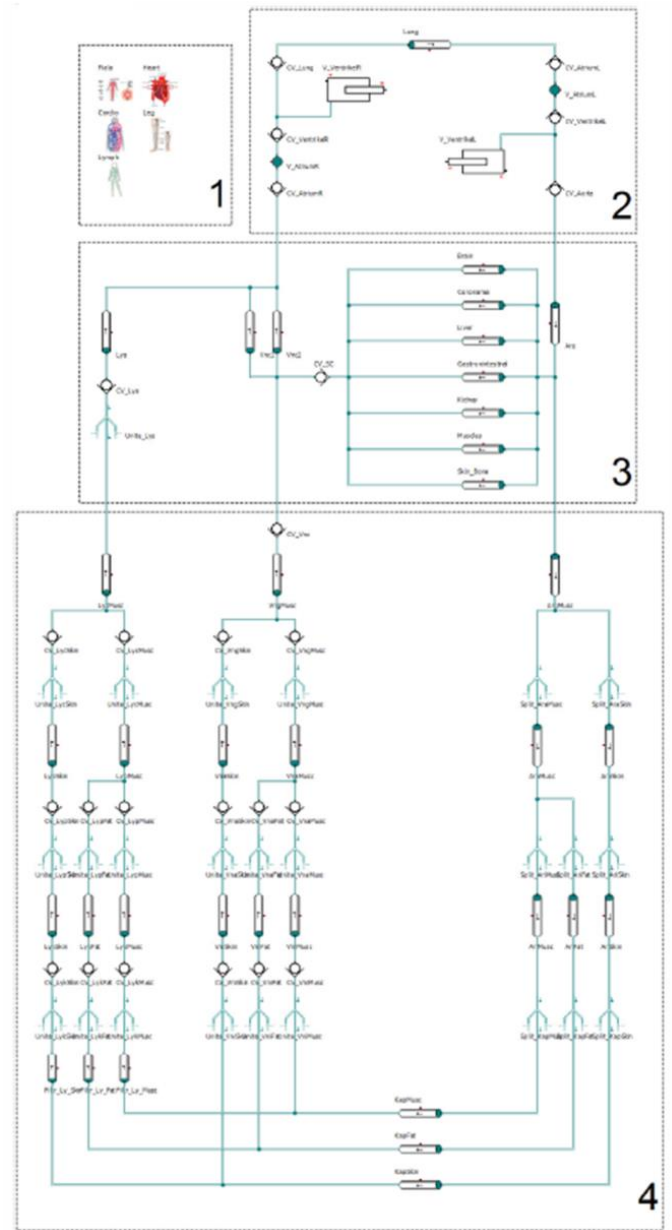

a) 1 types with parameters, 2 heart and lung, 3 organ systems and large blood vessels, 4 lower extremity.

Figure 5: Model of the decisive physiology of humans, (a) cardiovascular system, (b) tissue layers for heat conduction, (c) muscle pump to support the venous return flow.

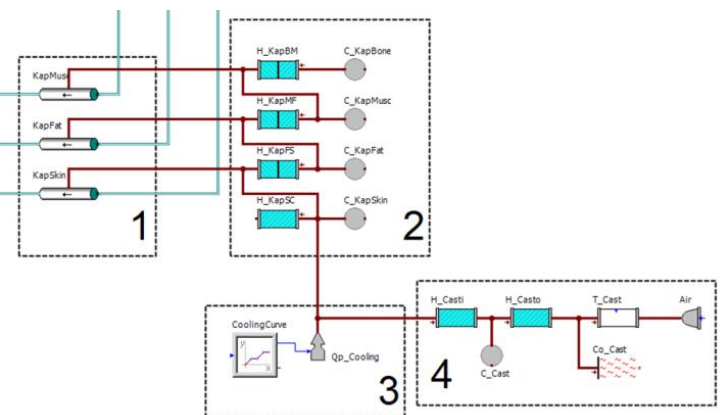

b) 1 blood vessels, 2 tissue layers, 3 interface to the cooling system, 4 cast and air.

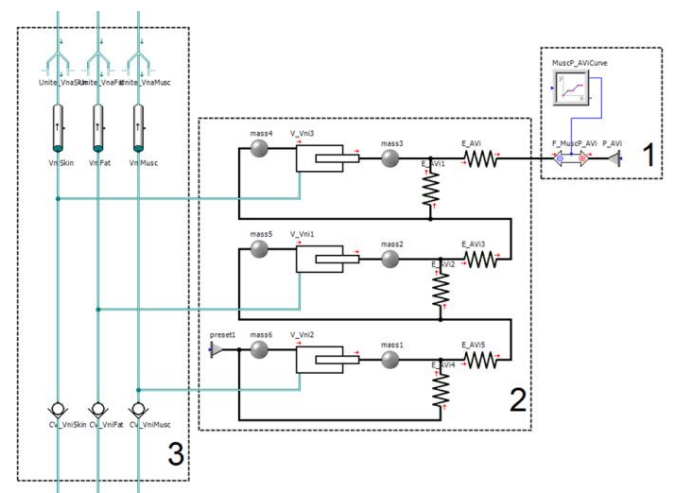

c) 1 force from the muscles, 2 force forwarding in the

elastic tissue, 3 blood vessels.
ans, (a) cardiovascular system, (b) tissue layers for heat 
Based on the results from the individual parts of the system, a basic design of a cooling and compression system is conceivable for the prevention or accelerated reduction of swellings of fractures. It is possible to deal with medically relevant problems, such as the compartment syndrome, or economically significant issues, such as long work absences.

\section{References}

[1] Bringezu G, Schreier O, Lehrbuch der Entstauungstherapie, Grundlagen, Beschreibung und Bewertung der Verfahren, Behandlungskonzepte für die Praxis, 4th edition, Heidelberg, Berlin: Springer-Verlag, 2014. ISBN 978-3-642-54921-2.

[2] Brunberg A, Autschbach R, Abel D, Ein objektorientierter Ansatz zur Modellierung des menschlichen Herz-Kreislauf-Systems, at Automatisierungstechnik, Vol. 56, No. 9, Oldenbourg Wissenschaftsverlag, pp. 476-482, 2008.

[3] CoolSystems Inc., Game Ready, 2017. URL: www.gameready.com (status: January 2017)

[4] DIN 33402-2, Ergonomie - Körpermaße des Menschen - Teil 2: Werte, 2005.

[5] Gioco Ltd., CRYO-2 Dynamic Cold Therapy, 2017. URL: www.giocosports.com (status: January 2017)

[6] Fahlke C, Linke W, Raßler B, Wiesner R, Taschenatlas Physiologie, 2nd edition, München: Urban \& Fischer Verlag, Elsevier GmbH, 2015.

[7] Fiala D, Dynamic Simulation of Human Heat Transfer and Thermal Comfort, Dissertation, Institute of Energy and Sustainable Development De Montfort University Leicester and Joseph-von-Egle Institut für angewandte Forschung FH Stuttgart Hochschule für Technik, 1998.

[8] Fulkerson EW, Egol KA, Timing Issues in Fracture Management: A Review of Current Concepts, Bulletin of NYU Hospital of Joint Diseases, Vol. 67, pp. 58-67, 2009.

[9] Hollensteiner B, Analgetische Wirkung einer Ganzkörperkältetherapie $-110^{\circ} \mathrm{C}, 3$ min, Dissertation, Medizinische Fakultät der Westfälischen Wilhelms-Universität Münster, 2003.

[10] Mateják M, Kulhánek T, Šilar J, Privitzer P, Ježek F, Kofránek J, Physiolibrary - Modelica library for Physiology, Proceedings of the 10th International Modelica Conference, Sweden, pp. 499-505, 2014.

[11] Meeusen R, Lievens P, The use of cryotherapy in sports injuries, Sports Medicine, Vol. 3, 1986.

[12] Ochs U, Winter E, Weise K, Malleolenfrakturen, Trauma und Berufskrankheit, Vol. 4, Springer Verlag, pp. 338-343, 2001.

[13] Paessler A, Nagel T, Combination of Gentle Local Hypothermia and Technical Lymphatic Drainage to Decrease Fracture Swellings, Proceedings of 2015 International Conference on Bio-Medical Engineering and Environmental Technology (BMEET-15), London, pp. 116-122, 2015.

[14] Paessler A, Nagel T, Lienig J, Physiology in fractures to optimize a cooling and compression bandage, Proceedings of ESI SimulationX User Forum, pp. 257-265, Dresden, 2016.

[15] Specht E, Der Mensch als wärmetechnisches System, 2005. URL: www.uni-magdeburg.de/isut/TV /Download/Der_Mensch_als_waermetechnisches_System.pdf (status: January 2017).

[16] Stöckle U, Hoffmann R, Schütz M, von Fournier C, Südkamp NP, Haas N, Fastest Reduction of Posttraumatic Edema: Continuous Cryotherapy or Intermittent Impulse Compression?, Foot \& Ankle International, American Orthopaedic Foot and Ankle Society, Vol. 18, No. 7, pp. 432-438, 1997.

\section{Disclosures}

This project is sponsored by the Federal Ministry for Economic Affairs and Energy and runs in cooperation with the MEDITECH Sachsen GmbH. The authors thank the University Center for Orthopaedics and Traumatology at the University Hospital Carl Gustav Carus in Dresden, especially Professor Dr. med. Hans Zwipp and Professor Dr. med. Stefan Rammelt, for providing medical information on fractures. 